\title{
Evaluation of a ketogenic diet for improvement of neurological recovery in individuals with acute spinal cord injury: a pilot, randomized safety and feasibility trial
}

\author{
Ceren Yarar-Fisher ${ }^{1} \cdot$ Adarsh Kulkarni $^{2} \cdot \mathrm{Jia} \mathrm{Li}^{1} \cdot$ Paige Farley $^{3} \cdot$ Cassandra Renfro $^{1} \cdot \mathrm{Hammad}$ Aslam ${ }^{1}$. \\ Patrick Bosarge ${ }^{4} \cdot$ Landon Wilson $^{5} \cdot$ Stephen Barnes ${ }^{5}$
}

Received: 29 June 2018 / Revised: 28 August 2018 / Accepted: 28 August 2018

(c) International Spinal Cord Society 2018

\begin{abstract}
Study design Longitudinal, randomized study.

Objectives (1) Test the safety and feasibility of a ketogenic diet (KD) intervention in the acute stages of spinal cord injury (SCI), (2) assess the effects of a KD on neurological recovery, and (3) identify potential serum biomarkers associated with KD-induced changes in neurological recovery.

Setting Acute care and rehabilitation facility.

Methods The KD is a high-fat, low-carbohydrate diet that includes $\approx 70-80 \%$ total energy as fat. Seven participants with acute complete and incomplete SCI (AIS A-D) were randomly assigned to KD $(n=4)$ or standard diet (SD, $n=3)$. Neurological examinations, resting energy expenditure analysis, and collection of blood for evaluation of circulating ketone levels were performed within $72 \mathrm{~h}$ of injury and before discharge. Untargeted metabolomics analysis was performed on serum samples to identify potential serum biomarkers that may explain differential responses between groups.

Results Our pilot findings primarily demonstrated that KD is safe and feasible to be administered in acute SCI. Furthermore, upper extremity motor scores were higher $(p<0.05)$ in the KD vs. SD group and an anti-inflammatory lysophospholipid, lysoPC 16:0, was present at higher levels, and an inflammatory blood protein, fibrinogen, was present at lower levels in the KD serum samples vs. SD serum samples.

Conclusion Taken together, these preliminary results suggest that a KD may have anti-inflammatory effects that may promote neuroprotection, resulting in improved neurological recovery in SCI. Future studies with larger sample size are warranted for demonstrating efficacy of KD for improving neurological recovery.
\end{abstract}

Ceren Yarar-Fisher

cyarar@uab.edu

1 Department of Physical Medicine and Rehabilitation, University of Alabama at Birmingham, Birmingham, AL 35294, USA

2 School of Medicine, University of Alabama at Birmingham, Birmingham, AL 35294, USA

3 Department of Emergency Medicine, University of Alabama at Birmingham, Birmingham, AL 35294, USA

4 Department of Surgery, University of Alabama at Birmingham, Birmingham, AL 35294, USA

5 Department of Pharmacology and Toxicology, University of Alabama at Birmingham, Birmingham, AL 35294, USA

\section{Introduction}

There is an urgent need for innovative therapies that improve neurological recovery following spinal cord injury (SCI). Despite extensive research, clinical advancements, and improved rehabilitation strategies, SCI continues to be a significant cause of disability and mortality [1-3]. After the initial injury, the damaged spinal cord undergoes multiple secondary pathological states that present important therapeutic targets for development of neuroprotective strategies for treatment of SCI.

A wide variety of evidence [4-10] suggests that the ketogenic diet (KD) may have beneficial disease-modifying effects in a broad range of neurological disorders characterized by hyperexcitability and death of neurons. The diet is composed of $80-90 \%$ fat, with carbohydrate and protein constituting the remainder of the nutrient intake. 
Energy is largely derived from oxidation of dietary fats. These fats are converted to the ketone bodies, $\beta$-hydroxybutyrate (BHB), acetoacetate, and acetone, which serve as an alternative energy source to glucose. Although the exact mechanism by which the diet provides neuroprotection is not fully understood, its effects on cellular energetics likely play a key role [10-13]. KDs increase circulating levels of ketone bodies, a more efficient fuel for the brain, resulting in enhanced capacity for energy production and an improved ability of neurons to resist negative metabolic challenges [14, 15]. Additionally, biochemical changes induced by the diet-including ketosis, high serum fat levels, and low serum glucose levels-may contribute to protection against neuronal death by apoptosis [16] and excitotoxicity [17] through a multitude of additional mechanisms, including antioxidant [12, 18] and antiinflammatory [19] actions. Neuroinflammation [20, 21], excitotoxicity [22], and apoptosis [23] are thought to be major mechanisms that contribute to secondary injury following SCI, thus protection against this sequelae via $\mathrm{KD}$ could produce a significant benefit in neurological recovery after SCI. A study showed that rats with cervical SCI showed a reduction in the lesion size and improved forelimb function when a KD was started $4 \mathrm{~h}$ after injury [24]. This effect was specific, as the neuroprotective effects could be ablated by treatment with a ketone transporter antagonist.

Emerging evidence also suggests that acute-phase hyperglycemia is a critical factor in the poor functional outcomes of SCI [25, 26]. The presence of hyperglycemia ( $\geq 126 \mathrm{mg} / \mathrm{dL}$ ) on hospital admission (irrespective of past diabetes mellitus history) was found to be strongly associated with a lower probability of improvement in motor and sensory function. Results of preclinical studies have indicated that hyperglycemia exacerbates inflammatory cytokine production, neural cell apoptosis, and demyelination during acute SCI in rodents [25]. These findings have shed light on the importance of achieving tight glycemic control in acute human SCI to obtain better neurological outcomes.

To extend these results, the aims of this pilot study were to (1) test the safety and feasibility of a KD intervention in an acute care setting (2), assess the effects of a KD on neurological recovery, and (3) employ untargeted metabolomics analysis to identify potential serum protein biomarkers associated with KD-induced changes in neurological recovery in individuals with acute SCI.

\section{Methods}

\section{Study design, protocol and participants}

Using a randomized, repeated-measures study design, seven participants ( $2 \mathrm{~F}$ and $5 \mathrm{M}, 35 \pm 12 \mathrm{y}$ ) with acute complete and incomplete (with anterior cord syndrome) SCI (American Spinal Injury Association Impairment Scale (AIS) A-D) were randomly assigned into one of the two study groups (KD and standard diet $[\mathrm{SD}]$ ) at a 1:1 ratio. The neurological level of injury (NLI) ranged from C4 to T11. As patients with Brown-Sequard and Central Cord Syndromes were shown to have more favorable prognoses [27, 28], they were not eligible for participation. Initially, there were two motor-complete (AIS A and B) and two motor-incomplete (AIS $\mathrm{C}$ ) patients in the $\mathrm{KD}$ group compared with one motor-complete (AIS A) and two motorincomplete (AIS C and D) patients in the SD group. Individual patient characteristics can be found in Table 1.

Patients were taken to acute care after injury. Neurological examinations, resting energy expenditure (REE) analysis, and blood collection were performed within $72 \mathrm{~h}$ of injury (after the initial trauma evaluation and resuscitation) and again before discharge from the rehabilitation hospital. Neurological tests were all performed by the same American Spinal Injury Association (ASIA) In-Step certified Physical Medicine and Rehabilitation clinician at baseline (at least $24 \mathrm{~h}$ after injury) and before discharge. The Sacral Sparing definition was used to define the completeness of injury [29]. Following acute care (16 \pm 9 days for KD vs. $16 \pm 6$ days for SD), patients were transferred to the Spain Rehabilitation Center (SRC) for in-patient rehabilitation care $(20 \pm 6$ days in KD vs. $18 \pm 1$ in SD). We certify that all applicable institutional and governmental regulations concerning the ethical use of human volunteers were followed during the course of this research.

\section{Interventions}

\section{Diet}

The KD was a high-fat, low-carbohydrate diet that included $\approx 72 \%$ total energy as fat, $\approx 25 \%$ as protein, and $\approx 3 \%$ as carbohydrate during enteral feeding and $\approx 65 \%$ total energy as fat, $\approx 27 \%$ as protein, and $\approx 8 \%$ as carbohydrate and fiber during solid feeding. The SD included $\approx 35 \%$ total energy as fat, $\approx 27 \%$ as protein, and $\approx 44 \%$ as carbohydrate and fiber. None of SD participants received enteral feeding. Dietary fat sources focused on animal and vegetable (for vegetarians) fats. Dietary protein sources included animal, plant, and nut and seed proteins, and dietary carbohydrate resources included vegetarian and vegan sources. $\mathrm{KetoCal}^{\circledR}$, a nutritionally complete, ready-to-feed ketogenic formula in a 3:1 ratio (fat:carbohydrate + protein), was provided by Nutricia for the patients who receive enteral nutrition. All semi-liquid and solid food were provided by the UAB Hospital Kitchen and Clinical Research Unit (CRU)'s Bionutrition Unit every day of the week. The overall energy amount was determined based on the REE, assessed via 
indirect calorimetry and multiplied by an activity factor. All patients in KD group adhered to the prescribed diet. Adherence to diet was closely monitored by the study team by measuring serum BHB levels once a week. Individual BHB data can be found in Table 1. Blood concentration of 0.5-3 mM (nutritional ketosis) was considered safe and acceptable. Adherence was also assessed using food records.

\section{In-patient rehabilitation care}

Patients in both groups underwent an intensive rehabilitation program (standard of care in the SRC). Therapies were offered 5.5 days/week for a total of $15 \mathrm{~h}$ per week. Rehabilitation care focused on respiratory therapy, passive and active range of motion, neuro-muscular re-education, mobility, transfers, wheelchair mobility skills, bowel and bladder management, tone and spasticity management, and skills for performing other activities of daily living.

\section{Clinical tests}

\section{Motor and sensory examinations}

Testing was performed according to the International Standards for the Neurological Classification of Spinal Cord Injury [30]. The average number of hours from injury to baseline (pre-intervention) measure was $67.8 \pm 5$. . The AIS Motor Index Score was used to measure motor function in the upper and lower extremities. For the sensory examination, each dermatome was tested for both sharp (pin-prick) and light-touch (LT) sensation and was graded on a threepoint scale. In addition, changes in neurological level of injury (NLI) and AIS classification were determined at discharge. One examiner performed all examinations and was blinded to the condition of the diet.

\section{Laboratory tests}

\section{Blood collection}

After a 10- to 12-h fast, blood samples were collected for measurement of serum glucose and lipid (cholesterol, triglycerides, high-density, and low-density lipoprotein) levels at baseline (within 72-h injury) and discharge. Serum glucose and lipids were analyzed using an automated glucose analyzer (Sirrus analyzer; Stanbio Laboratory; Boerne, TX, USA) as per manufacturer's instructions. Lipid analysis was done on six patients due to limited amount of sampled blood in the SD group. In addition, blood samples were collected for measurement of blood ketone (BHB) levels once a week, $2 \mathrm{~h}$ after first meal of the day. An automated analyzer (Sirrus analyzer; Stanbio Laboratory; Boerne, TX) 
was used to confirm nutritionally induced ketosis (blood BHB concentration, $0.5-3 \mathrm{mM}$ ) in the $\mathrm{KD}$ group.

\section{Untargeted metabolomics analysis}

As an adjunct or alternative to neurological assessments, we have performed an untargeted metabolomics via liquid chromatography-mass spectrometry (LC-MS)/MS global analysis on serum samples to identify potential biomarkers that can predict neurological recovery and explain the differences between groups. LC-MS/MS analysis and data processing (via MetaboAnalyst-www.metaboanalyst.ca and METLIN—http://metlin.scripps.edu) have been performed at UAB Targeted Metabolomics and Proteomics Laboratory with established procedures published elsewhere [31-33].

\section{Statistical analyses}

Statistical analyses were performed using SAS, version 9.4 (SAS Institute, Inc.; Cary, NC). Mixed-model, repeatedmeasures analysis of variance was used to assess the effects of group (KD, SD), time (pre-, post-intervention), and the group-by-time interaction on the outcomes of interest (motor and sensory scores and peak intensities of variable importance in projection [VIP] scores from the metabolomics analysis). A compound symmetry covariance matrix was assumed for analyses. Due to the small sample size and pilot nature of the current study, post hoc comparisons were performed without adjustment for multiple pairwise comparisons. Statistical tests were two-sided, and $p$-values $<0.05$ were considered statistically significant.

\section{Results}

\section{BHB, glucose and lipid concentrations}

As expected, the KD group maintained nutritional ketosis (Table 1) over $5 \pm 2$ weeks (KD BHB concentration: $1.02 \mathrm{mmol} / \mathrm{L}$ vs. $0.1 \mathrm{mmol} / \mathrm{L}$ in the $\mathrm{SD}$ group). Individual BHB (mmol/L), fasting glucose, and lipid $(\mathrm{mg} / \mathrm{dL})$ levels are shown in Table 1. KD group demonstrated a reduction in fasting glucose (Table 1, $\Delta: 24 \mathrm{mg} / \mathrm{dL}$; pre: 119.5 vs. post: $95.25 \mathrm{mg} / \mathrm{dL}$ ). No change in fasting glucose was found in the SD group. In addition, KD group maintained a normal lipid profile in contrast to SD group, which showed an increase in cholesterol, triglycerides, and low-density lipoprotein at discharge.

\section{Motor and sensory examinations}

In $86 \%$ of our patients ( 5 of 7 ), there was no change in NLI between the initial assessment (within $72 \mathrm{~h}$ of injury) and at discharge ( $5 \pm 2$ weeks post-injury). Two patients in the KD group demonstrated improvements in the NLI and AIS classification (T10 AIS A to T11 AIS B and C4 AIS C to C5 AIS D). Individual upper and lower-extremity motor scores (UEMSs and LEMSs), LT and pin-prick sensory scores at baseline and discharge are shown in Fig. 1a-d. An overall time effect was observed for UEMS $(p<0.05)$. The KD group demonstrated an increase $(p<0.05)$ in UEMS compared with baseline scores (KD: 24 [pre] vs. 38.7 [post]), whereas no change $(p=0.3)$ was observed in the SD group (SD: 39.5 [pre] vs. 47 [post]). Between the baseline and discharge timepoints, 15 UEMS and 13 LEMS points were recovered in the KD group vs. 8 UEMS and 6 LEMS points recovered in the SD group. In addition, no effect of time and group was observed in sensory outcomes (LT or pin-prick scores).

\section{Untargeted metabolomics analysis}

Using XCMSonline, peaks containing individual ions were aligned according to their retention times. Multivariate analysis (unsupervised principal component analysis) and supervised partial least squares-discriminant analysis (PLSDA) were performed to assess chemometric separation among the SD and KD samples. PLS-DA showed a separation of the control and KD groups (Fig. 2a, b). Identified by VIP analysis as ions most contributing to the separations seen in Fig. 2a, b were characterized by having high $m / z$ values (600-800) and being doubly charged ions, thereby suggesting masses in the 1200-1600 Da range. These ions and their MS/MS spectra were analyzed using Protein Pilot ${ }^{\mathrm{TM}}$, which revealed that they were fibrinogen-related peptides (Fibrinogen alpha and beta subunits) (Table 2). Compared with the SD, contents of these peptides were decreased in the KD group. Inspection of the remaining ions in the VIP data using the METLIN database revealed the presence of lysophophatidylcholine 16:0 (LysoPC 16:0). Compared with the SD, contents of LysoPC was increased in the KD group.

\section{Discussion}

The primary finding of this pilot project is that a KD intervention was safe and feasible to be administered in the acute care setting for individuals with SCI as nutritional ketosis and normoglycemia were safely achieved, normal lipid levels were maintained, and no adverse effects were noted. Secondarily, UEMSs were higher $(p<0.05)$ in the KD group compared with the SD group. In addition, levels of fibrinogen, a pleiotropic blood protein that is known to regulate neuroinflammation, were lower, and levels of lysoPC 16:0, an anti-inflammatory lysophospholipid, were higher in the KD group compared with the SD group. 

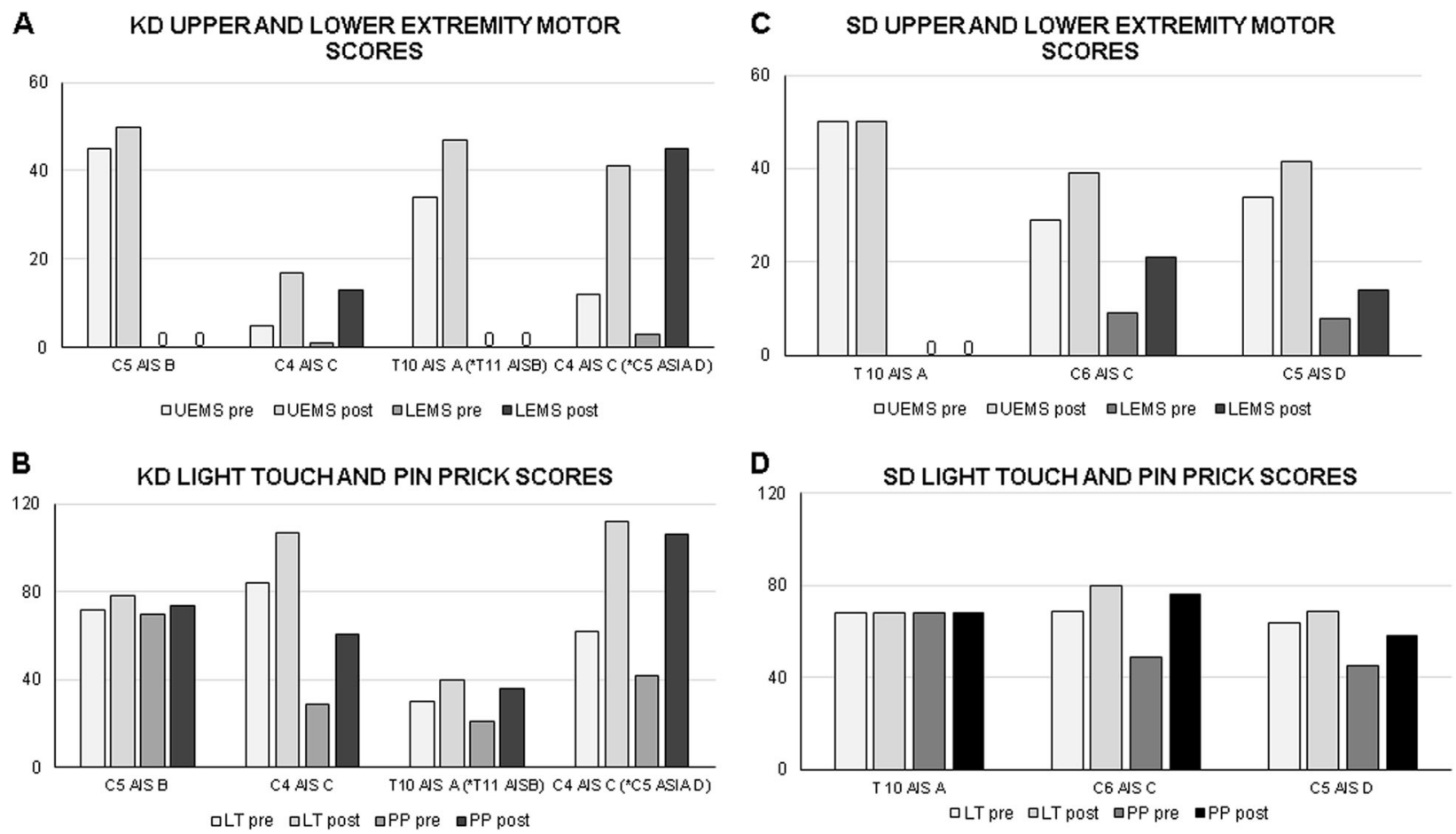

Fig. 1 Effects of the ketogenic diet (KD) vs. standard diet (SD) on motor and sensory scores after spinal cord injury. Individual upper extremity motor scores (UEMSs) and lower-extremity motor scores

(LEMSs) for KD (a) and SD (b) group; and light-touch (LT) and pinprick (PP) sensory scores for KD (c) and SD (d) group. *Indicates AIS grade conversion

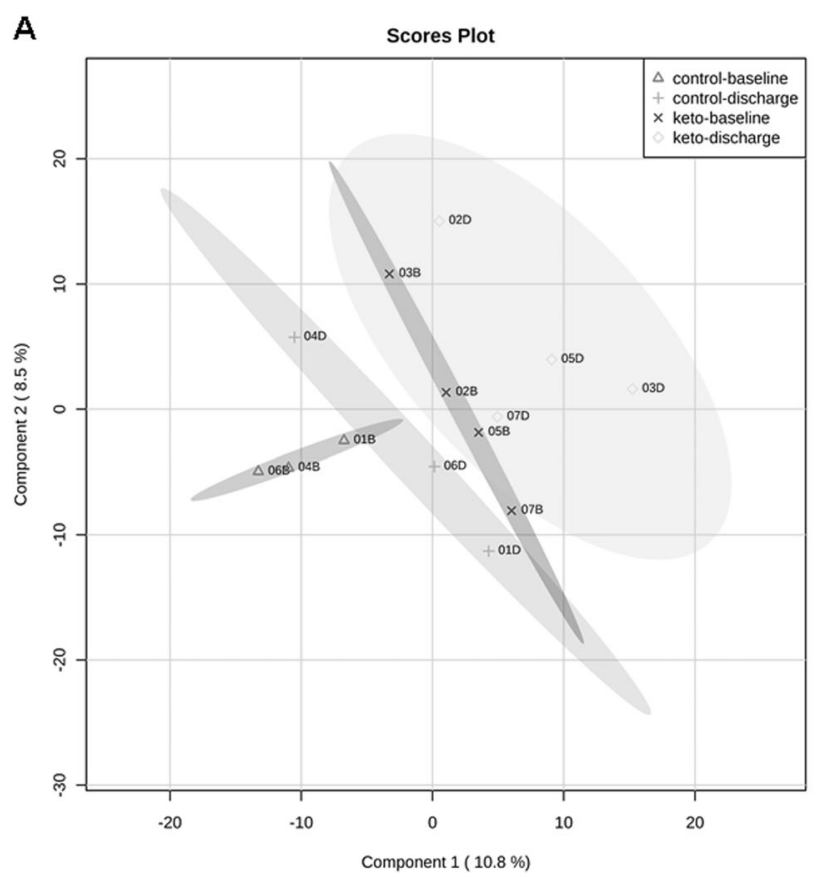

Fig. 2 Partial least squares-discriminant analysis (PLS-DA) scores plot from the LC-MDS metabolomics data collected in negative (a) and positive ion (b) modes between selected principal components 1 and 2 in the ketogenic diet (KD, patient codes: \#02, 03, 06, and 07) vs. standard diet (SD, patient codes: \#01, 04, and 05) group data. Each

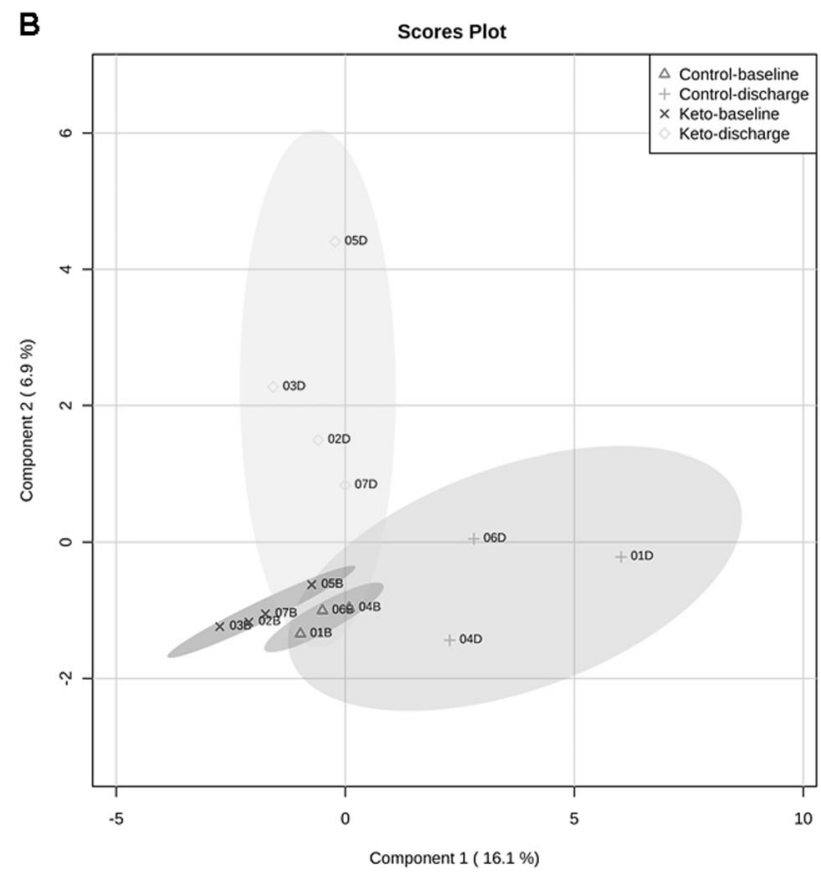

component is the sum of the contributions made by each metabolite ion to it. The ellipses represent the $95 \%$ confidence limits for each group (red-baseline (B) standard diet, green—discharge (D) standard diet, blue — baseline (B) keto diet, light blue_-discharge (D) 
Table 2 Variable importance in projection (VIP) features identified by PLS-DA in positive and negative ion mode

\begin{tabular}{lll}
\hline VIP features & Peptide sequence/chemical formula & $\mathrm{m} / \mathrm{z}$ mode $(+/-)$ \\
\hline Fibrinogen alpha subunit & DSGEGDFLAEGGGVR & $733.831(+)$ \\
Fibrinogen alpha subunit & ADSGEGDFLAEGGGVR & $768.850(+)$ \\
Fibrinogen beta subunit & Pyro-QGVNDNEEGFFSA & $698.786(+)$ \\
Fibrinogen beta subunit & Pyro-QGVNDNEEGFF & $619.751(+)$ \\
Fibrinogen alpha subunit & DSGEGDFLAEGGGVR & $731.351(-)$ \\
Fibrinogen alpha subunit & SGEGDFLAEGGGVR & $673.824(-)$ \\
Fibrinogen beta subunit & Pyro-QGVNDNEEGFF & $617.736(-)$ \\
Fibrinogen beta subunit & QGVNDNEEGFFS & $661.269(-)$ \\
LysoPC $(16: 0)$ & $\mathrm{C}_{24} \mathrm{H}_{50} \mathrm{NO}_{7} \mathrm{P}$ & $496.340(+)$ \\
\hline
\end{tabular}

D) groups despite randomization. Patients with AIS C have been shown to gain more points compared with other AIS grades. Consistent with this finding, one subject who was an AIS D in SD gained fewer points than those with AIS grade $\mathrm{C}$ in KD group due to a possible ceiling effect in AIS D injuries. In addition, the International Standards for the Neurological Classification of Spinal Cord Injury assessment tool has practical limitations. It may be difficult to perform an accurate neurological test due to sedation or pain after surgery or due to pain caused by chronic conditions such as carpal tunnel syndrome. In the present study, none of the patients were sedated or in pain, and all patients were fully cognizant during motor and sensory examinations, minimizing these confounders. However, one patient in the KD group (Table 1, patient code: \#02) presented with carpal tunnel syndrome at admission. Although carpal tunnel syndrome was not treated during rehabilitation care, it may have impacted the patient's performance during neurological tests at baseline and discharge due to weakness in hand and fingers. In addition, examiner effects may be introduced if more than one examiner performs neurological testing. One certified examiner conducted all assessments over the course of the study to eliminate examiner effects.

To gain further insight into the mechanisms that underlay the differential neurological recovery between the $\mathrm{KD}$ and SD groups, we performed an untargeted metabolomics analysis to identify potential serum biomarkers associated with changes in neurological recovery. Although the intent of the metabolomics analysis was to detect small molecule compounds that are part of metabolic pathways, in this case the most significant VIP ions had masses $(\mathrm{m} / \mathrm{z})$ that were above 600 . When examined more closely, these ions had 1-13C-ions that were spaced at $0.5 \mathrm{~m} / \mathrm{z}$, i.e., they were doubly charged. Their MSMS spectra suggested that they were peptides. Analysis with the proteomics software (Protein Pilot ${ }^{\mathrm{TM}}$ ) confirmed this and further revealed that each peptide (see in the Table 2) was derived from proteins, fibrinogen (alpha and beta subunits) and lysoPC 16:0. Furthermore, not only were positively charged peptide ions detected, but also their negatively charged counterparts. 
Although we can only speculate as to the mechanisms connected to these observations, it is possible that low fibrinogen and high lysoPC 16:0 levels in the KD group may have promoted neuroprotection, thus improving neurological recovery. There is abundant evidence [39-41] that fibrinogen is present in the nervous system after traumatic injuries characterized by vascular rupture or disruption of the blood-brain barrier in the central nervous system. Rupture of the vasculature allows entry of blood proteins into nervous system tissue, which is shortly followed by edema and neural damage. Fibrinogen interacts with cell surface receptors expressed by microglia, and activation of microglia by fibrinogen has been shown to initiate neuroinflammation at sites of vascular injury. In addition, growing evidence [42-44] suggests that lysoPCs can reduce systemic inflammation in rodent models of sepsis and ischemia, as well as human chronic inflammatory disorders like ulcerative colitis. Under conditions of severe inflammatory stress, activity of plasma phospholipase A2 (sPLA2), an enzyme associated with the activity of the innate immune system and inflammatory disorders, increases. Systemic lysoPCs help to shift the balance back toward sPLA2 inhibition and cytoprotection. Thus, taken together, these results suggest that anti-inflammatory effects of the KD, such as decreased fibrinogen and increased lysoPC 16:0 levels, may have promoted neuroprotection, thereby improving neurological recovery. Our findings are consistent with previous work in animals demonstrating a potential link between $\mathrm{KD}$, anti-inflammatory mechanisms in the central nervous system, and modification of the progression of neurological disease. For example, the high fatty acid load associated with a KD has been shown to activate peroxisome proliferator-activated receptor $\alpha$, which may, in turn, have inhibitory effects on the proinflammatory transcription factors nuclear factor- $\mathrm{\kappa B}$ and activation protein-1 [19].

Emerging evidence suggests that acute-phase hyperglycemia is a critical factor in the poor functional outcomes of SCI $[25,26]$. Surprisingly, enteral and solid diets in SCI acute and sub-acute care have traditionally promoted high carbohydrate nutritional content (carbohydrate: 45\%; fat: $30 \%$, and protein: $25 \%$ ). The presence of hyperglycemia on hospital admission (irrespective of past diabetes mellitus history) was found to be strongly associated with a lower probability of improvement in neurological function [25]. The ability of a KD to ameliorate the diabetic state and help stabilize hyperglycemia has been repeatedly shown in human studies $[45,46]$. Consistent with this literature, there was an overall reduction in fasting glucose in the KD group. One patient (Table 1, patient code: \#07) admitted with hyperglycemia (fasting glucose: $178 \mathrm{mg} / \mathrm{dL}$ ) achieved normoglycemia (fasting glucose: $115 \mathrm{mg} / \mathrm{dL}$ ) following the KD intervention. The results of our study suggest that the KD may be safe to administer in patients with hyperglycemia and promote a normal glycemic state following SCI.
The greatest limitation of our study is the small sample size. We are aware that this pilot study is insufficiently powered to detect minimal, but clinically important differences, as well as other potential biomarkers reflective of neurological recovery. In addition to the treatment under investigation, the primary outcome variables may have been influenced by other covariates, such as NLI, severity of injury, sex, age, and rehabilitative therapies and that cannot be accounted for in the present study due to the small sample size. Future studies with larger sample size are warranted for demonstrating efficacy of KD for improving neurological recovery in the acute stages of SCI.

Acknowledgements The authors sincerely thank the participants and their families for their tireless dedication. We also thank Rhonda Pierce, RD, Margaret Peoples, RD, and the UAB Bionutrition Kitchen for development and delivery of KDs, as well as the UAB Department of Emergency Medicine Research Assistant Program for their assistance with study coordination and data collection.

Funding This work was supported by KL2TR001419-01 (CY-F) and UAB Center for Clinical and Translational Science (UL1-TR-001417).

\section{Compliance with ethical standards}

Conflict of interest The authors declare that they have no conflict of interest.

\section{References}

1. DeVivo MJ, Krause JS, Lammertse DP. Recent trends in mortality and causes of death among persons with spinal cord injury. Arch Phys Med Rehabil. 1999;80:1411-9.

2. Jensen MP, Molton IR, Groah SL, Campbell ML, Charlifue S, Chiodo A, et al. Secondary health conditions in individuals aging with SCI: terminology, concepts and analytic approaches. Spinal Cord. 2012;50:373-8.

3. Strauss DJ, Devivo MJ, Paculdo DR, Shavelle RM. Trends in life expectancy after spinal cord injury. Arch Phys Med Rehabil. 2006;87:1079-85.

4. Gasior M, Rogawski MA, Hartman AL. Neuroprotective and disease-modifying effects of the ketogenic diet. Behav Pharmacol. 2006;17:431-9.

5. Prins ML, Matsumoto JH. The collective therapeutic potential of cerebral ketone metabolism in traumatic brain injury. J Lipid Res. 2014:55:2450-7.

6. Vanitallie TB, Nonas C, Di Rocco A, Boyar K, Hyams K, Heymsfield SB. Treatment of Parkinson disease with diet-induced hyperketonemia: a feasibility study. Neurology. 2005;64:728-30.

7. Freeman J, Veggiotti P, Lanzi G, Tagliabue A, Perucca E, Institute of Neurology ICMF. The ketogenic diet: from molecular mechanisms to clinical effects. Epilepsy Res. 2006;68:145-80.

8. Freeman JM, Vining EP, Pillas DJ, Pyzik PL, Casey JC, Kelly LM. The efficacy of the ketogenic diet-1998: a prospective evaluation of intervention in 150 children. Pediatrics. 1998;102:1358-63.

9. Reger MA, Henderson ST, Hale C, Cholerton B, Baker LD, Watson GS, et al. Effects of beta-hydroxybutyrate on cognition in memory-impaired adults. Neurobiol Aging. 2004;25:311-4. 
10. Veyrat-Durebex C, Reynier P, Procaccio V, Hergesheimer R, Corcia P, Andres CR, et al. How can a ketogenic diet improve motor function? Front Mol Neurosci. 2018;11:15.

11. Bough KJ, Gudi K, Han FT, Rathod AH, Eagles DA. An anticonvulsant profile of the ketogenic diet in the rat. Epilepsy Res. 2002;50:313-25.

12. Veech RL. The therapeutic implications of ketone bodies: the effects of ketone bodies in pathological conditions: ketosis, ketogenic diet, redox states, insulin resistance, and mitochondrial metabolism. Prostaglandins Leukot Essent Fat Acids. 2004;70:309-19.

13. Veech RL, Chance B, Kashiwaya Y, Lardy HA, Cahill GF Jr. Ketone bodies, potential therapeutic uses. IUBMB Life. 2001;51:241-7.

14. Kashiwaya Y, Takeshima T, Mori N, Nakashima K, Clarke K, Veech RL. D-beta-hydroxybutyrate protects neurons in models of Alzheimer's and Parkinson's disease. Proc Natl Acad Sci USA. 2000;97:5440-4.

15. Tieu K, Perier C, Caspersen C, Teismann P, Wu DC, Yan SD, et al. D-beta-hydroxybutyrate rescues mitochondrial respiration and mitigates features of Parkinson disease. J Clin Invest. 2003;112:892-901.

16. Noh HS, Kim DW, Kang SS, Cho GJ, Choi WS. Ketogenic diet prevents clusterin accumulation induced by kainic acid in the hippocampus of male ICR mice. Brain Res. 2005;1042:114-8.

17. Noh HS, Hah YS, Nilufar R, Han J, Bong JH, Kang SS, et al. Acetoacetate protects neuronal cells from oxidative glutamate toxicity. J Neurosci Res. 2006;83:702-9.

18. Ziegler DR, Ribeiro LC, Hagenn M, Siqueira IR, Araujo E, Torres IL, et al. Ketogenic diet increases glutathione peroxidase activity in rat hippocampus. Neurochem Res. 2003;28:1793-7.

19. Cullingford TE. The ketogenic diet; fatty acids, fatty acidactivated receptors and neurological disorders. Prostaglandins Leukot Essent Fat Acids. 2004;70:253-64.

20. Mabon PJ, Weaver LC, Dekaban GA. Inhibition of monocyte/ macrophage migration to a spinal cord injury site by an antibody to the integrin alphaD: a potential new anti-inflammatory treatment. Exp Neurol. 2000;166:52-64.

21. Popovich PG, Wei P, Stokes BT. Cellular inflammatory response after spinal cord injury in Sprague-Dawley and Lewis rats. J Comp Neurol. 1997;377:443-64.

22. Farooque M, Hillered L, Holtz A, Olsson Y. Changes of extracellular levels of amino acids after graded compression trauma to the spinal cord: an experimental study in the rat using microdialysis. J Neurotrauma. 1996;13:537-48.

23. Beattie MS, Farooqui AA, Bresnahan JC. Review of current evidence for apoptosis after spinal cord injury. J Neurotrauma. 2000;17:915-25.

24. Streijger F, Plunet WT, Lee JH, Liu J, Lam CK, Park S, et al. Ketogenic diet improves forelimb motor function after spinal cord injury in rodents. PLoS ONE. 2013;8:e78765.

25. Kobayakawa K, Kumamaru H, Saiwai H, Kubota K, Ohkawa Y, Kishimoto $\mathrm{J}$, et al. Acute hyperglycemia impairs functional improvement after spinal cord injury in mice and humans. Sci Transl Med. 2014;6:256ra137.

26. Sala F, Menna G, Bricolo A, Young W. Role of glycemia in acute spinal cord injury. Data from a rat experimental model and clinical experience. Ann N Y Acad Sci. 1999;890:133-54.

27. Kirshblum SC, O'Connor KC. Predicting neurologic recovery in traumatic cervical spinal cord injury. Arch Phys Med Rehabil. 1998;79:1456-66.

28. Pollard ME, Apple DF. Factors associated with improved neurologic outcomes in patients with incomplete tetraplegia. Spine (Phila PA 1976). 2003;28:33-9.
29. Waters RL, Adkins RH, Yakura JS. Definition of complete spinal cord injury. Paraplegia. 1991;29:573-81.

30. Ditunno JF Jr., Young W, Donovan WH, Creasey G. The international standards booklet for neurological and functional classification of spinal cord injury. American Spinal Injury Association. Paraplegia. 1994;32:70-80.

31. Prasain JK, Wilson LS, Arabshahi A, Grubbs C, Barnes S. Mass spectrometric evidence for the modification of small molecules in a cobalt-60-irradiated rodent diet. J Mass Spectrom. 2017; 52:707.

32. Barnes S, Benton HP, Casazza K, Cooper SJ, Cui X, Du X, et al. Training in metabolomics research. II. Processing and statistical analysis of metabolomics data, metabolite identification, pathway analysis, applications of metabolomics and its future. J Mass Spectrom. 2016;51:535-48.

33. Barnes S, Benton HP, Casazza K, Cooper SJ, Cui X, Du X, et al. Training in metabolomics research. I. Designing the experiment, collecting and extracting samples and generating metabolomics data. J Mass Spectrom. 2016;51:461-75.

34. Marino RJ, Burns S, Graves DE, Leiby BE, Kirshblum S, Lammertse DP. Upper- and lower-extremity motor recovery after traumatic cervical spinal cord injury: an update from the national spinal cord injury database. Arch Phys Med Rehabil. 2011;92:369-75.

35. Steeves JD, Kramer JK, Fawcett JW, Cragg J, Lammertse DP, Blight AR, et al. Extent of spontaneous motor recovery after traumatic cervical sensorimotor complete spinal cord injury. Spinal Cord. 2011;49:257-65.

36. Waters RL, Adkins RH, Yakura JS, Sie I. Motor and sensory recovery following complete tetraplegia. Arch Phys Med Rehabil. 1993;74:242-7.

37. Waters RL, Adkins RH, Yakura JS, Sie I. Motor and sensory recovery following incomplete tetraplegia. Arch Phys Med Rehabil. 1994;75:306-11.

38. Waters RL, Adkins RH, Yakura JS, Sie I. Motor and sensory recovery following incomplete paraplegia. Arch Phys Med Rehabil. 1994;75:67-72.

39. Bardehle S, Rafalski VA, Akassoglou K. Breaking boundariescoagulation and fibrinolysis at the neurovascular interface. Front Cell Neurosci. 2015;9:354.

40. Davalos D, Baeten KM, Whitney MA, Mullins ES, Friedman B, Olson ES, et al. Early detection of thrombin activity in neuroinflammatory disease. Ann Neurol. 2014;75:303-8.

41. Ryu JK, Davalos D, Akassoglou K. Fibrinogen signal transduction in the nervous system. J Thromb Haemost. 2009;7(Suppl 1):151-4.

42. Cunningham TJ, Yao L, Lucena A. Product inhibition of secreted phospholipase A2 may explain lysophosphatidylcholines' unexpected therapeutic properties. J Inflamm (Lond). 2008;5:17.

43. Treede I, Braun A, Sparla R, Kuhnel M, Giese T, Turner JR, et al. Anti-inflammatory effects of phosphatidylcholine. J Biol Chem. 2007;282:27155-64.

44. Triggiani M, Granata F, Frattini A, Marone G. Activation of human inflammatory cells by secreted phospholipases A2. Biochim Biophys Acta. 2006;1761:1289-300.

45. Hussain TA, Mathew TC, Dashti AA, Asfar S, Al-Zaid N, Dashti HM. Effect of low-calorie versus low-carbohydrate ketogenic diet in type 2 diabetes. Nutrition. 2012;28:1016-21.

46. Feinman RD, Pogozelski WK, Astrup A, Bernstein RK, Fine EJ, Westman EC, et al. Dietary carbohydrate restriction as the first approach in diabetes management: critical review and evidence base. Nutrition. 2015;31:1-13. 